\title{
NODAL SETS FOR EIGENFUNCTIONS OF THE LAPLACIAN ON SURFACES
}

\author{
HAROLD DONNELLY AND CHARLES FEFFERMAN
}

\section{INTRODUCTION}

Let $M^{2}$ be a compact connected surface having a smooth Riemannian metric. The associated Laplacian $\Delta$ is a negative definite selfadjoint elliptic operator. Suppose that $F$ is a real eigenfunction, $\Delta F=-\lambda F$. If $M$ has a nonempty boundary, we require Dirichlet or Neumann boundary conditions for $F$.

The nodal set $N$ of $F$ is simply the zero set of $F$. It contains a finite number of singular points $S=\{x \in M \mid F(x)=0$ and $\nabla F(x)=0\}$. Let $B\left(p, a_{1} \lambda^{-1 / 4}\right)$ be a geodesic ball in $M$ centered at $p$ with radius $a_{1} \lambda^{-1 / 4}$. Here $a_{1}$ is a suitable constant. Our first result is to count the number of singular points up to multiplicity in such a ball.

Theorem 1.1. Assume that $p_{\nu} \in S \cap B\left(p, a_{1} \lambda^{-1 / 4}\right)$ are singular points where $F$ vanishes to order $n_{\nu}+1$. Then $\sum n_{\nu} \leq a_{2} \lambda^{1 / 2}$.

Of course, we say that $F$ vanishes to order $n_{\nu}+1$ if the first nonvanishing term in its Taylor expansion is of order $n_{\nu}+1$. Since $p_{\nu} \in S$, we have $n_{\nu} \geq 1$.

In our earlier paper [5], we showed that $F$ can vanish at a single point to at most order $a_{2} \lambda^{1 / 2}$. This result represented a quantitative version of the classical unique continuation theorem by Aronszajn [1]. The order $a_{2} \lambda^{1 / 2}$ of vanishing is achieved at isolated single points by spherical harmonics on $S^{2}$. Another example, described below, shows that $B\left(p, a_{1} \lambda^{-1 / 4}\right)$ cannot be replaced in Theorem 1.1 by any bigger $B\left(p, a_{1} \lambda^{-\varepsilon}\right)$ with $\varepsilon<\frac{1}{4}$ while still maintaining the upper bound $a_{2} \lambda^{1 / 2}$.

Now consider the one-dimensional Hausdorff measure $\mathscr{H}^{1} N$ of the nodal set $N$. Since $N-S$ is a one-dimensional manifold and $S$ is a finite set, one has $\mathscr{H}^{1} N=\mathscr{H}^{1}(N-S)$. Our second main result is

Theorem 1.2. $\mathscr{H}^{1}\left(N \cap B\left(p, a_{3} \lambda^{-1 / 4}\right)\right) \leq a_{4} \lambda^{1 / 4}$.

The estimate $a_{4} \lambda^{1 / 4}$ is the optimal upper bound for the nodal set lying in a ball with radius $a_{3} \lambda^{-1 / 4}$. This reflects the fact that $F$ may vanish to order

Received by the editors January 13, 1989.

1980 Mathematics Subject Classification (1985 Revision). Primary 58G25. 
$a_{2} \lambda^{1 / 2}$ at isolated singular points. We do not know if the radius $a_{3} \lambda^{-1 / 4}$ of Theorem 1.2 can be replaced by any $a_{3} \lambda^{-\varepsilon}$, where $\varepsilon<\frac{1}{4}$, to give an upper bound $\mathscr{H}^{1}\left(N \cap B\left(p, a_{3} \lambda^{-\varepsilon}\right)\right)<a_{4} \lambda^{1 / 2-\varepsilon}$. However, both of our theorems follow from certain basic Carleman inequalities. Since the first theorem is sharp, there appears to be a serious obstruction to improving the second theorem via the current techniques.

Covering $M$ by order $\lambda^{1 / 2}$ balls of radius $a_{3} \lambda^{-1 / 4}$, we immediately deduce

Corollary 1.3. $\mathscr{H}^{1} N<a_{5} \lambda^{3 / 4}$.

Yau has conjectured that $\mathscr{H}^{1} N<a_{6} \lambda^{1 / 2}$. We proved this conjecture [5] when $M$ is a real analytic manifold with real analytic metric. For differentiable manifolds, Hardt and Simon [8] established the estimate $\mathscr{H}^{1} N<a_{7} \exp \left(a_{8} \sqrt{\lambda} \log \lambda\right)$. In particular, Corollary 1.3 gives an upper bound of polynomial growth. Some time ago [2], Brüning derived the complementary lower bound $\mathscr{H}^{1} N>a_{9} \lambda^{1 / 2}$ in Yau's conjecture.

Recently, Nadirashvili [10] announced the upper bound

$$
\mathscr{H}^{1} N<a_{10} \lambda \log (1+\lambda) .
$$

A brief sketch of the proof was provided. In the case when $M$ is only differentiable, his method seems substantially different from ours.

\section{Carleman INequalities}

In his seminal work [3] on unique continuation for first-order differential equations in two independent variables, Carleman derived certain weighted integral inequalities. His method of proof relied upon integral representations via fundamental solutions. We develop related inequalities using a rather different approach, which involves repeated partial integration and estimates of commutators. These commutator functions may be interpreted as the Gaussian curvatures of conformally flat metrics. Consequently, our estimates are reminiscent of the standard Bochner formulas [7]. However, we eschew this differential geometry viewpoint in favor of a more elementary outlook.

Consider the weighted Hilbert space $L^{1}\left(\mathscr{D}, e^{-\phi} d x d y\right)$ of complex-valued functions. Here $\mathscr{D}$ is a bounded open subset of the complex plane, and $\phi$ is a smooth real-valued function. Our primary concern involves smooth compactly supported functions $u \in C_{0}^{\infty}(\mathscr{D})$, a subset of $L^{2}\left(\mathscr{D}, e^{-\phi} d x d y\right)$. One has the basic first-order differential operators $\partial u=\partial u / \partial z=\frac{1}{2}(\partial / \partial x-i \partial / \partial y) u$ and its companion $\bar{\partial} u=\partial u / \partial \bar{z}=\frac{1}{2}(\partial / \partial x+i \partial / \partial y) u$. In our weighted Hilbert space, the adjoint of $\bar{\partial}$ is $\bar{\partial}^{*}$. A calculation verifies the formula $\bar{\partial}^{*} v=-e^{\phi} \partial\left(e^{-\phi} v\right)$.

A priori, the commutator $\left[\bar{\partial}, \bar{\partial}^{*}\right]$ might only be expected to be a first-order operator. However, we compute that $\left[\bar{\partial}, \bar{\partial}^{*}\right]$ is simply the zeroth order multiplication operator $\left[\bar{\partial}, \bar{\partial}^{*}\right] u=(\bar{\partial} \partial \phi) u=\left(\frac{1}{4} \Delta_{0} \phi\right) u$. Here $\Delta_{0}$ denotes the Euclidean Laplacian. The simplicity of this commutator facilitates the proof of 
Proposition 2.1. Let $\Phi$ be a smooth positive function defined on a bounded open set $\mathscr{D}$ of the complex plane. If $u \in C_{0}^{\infty}(\mathscr{D})$ is complex valued, then

$$
\int_{\mathscr{D}}|\bar{\partial} u|^{2} \Phi \geq \int_{\mathscr{D}} \frac{1}{4}\left(\Delta_{0} \ln \Phi\right)|u|^{2} \Phi
$$

Here the integral is with respect to Lebesgue measure.

Proof. We define $\phi=-\ln \Phi$ or equivalently $\Phi=e^{-\phi}$. Working in the weighted Hilbert space $L^{2}\left(\mathscr{D}, e^{-\phi} d x d y\right)$ we observe that

$$
\begin{aligned}
0 \leq\left\|\bar{\partial}^{*} u\right\|^{2} & =\left\langle\bar{\partial} \bar{\partial}^{*} u, u\right\rangle=\left\langle\bar{\partial}^{*} \bar{\partial} u, u\right\rangle+\left\langle\left[\bar{\partial}, \bar{\partial}^{*}\right] u, u\right\rangle \\
& =\|\bar{\partial} u\|^{2}+\left\langle\frac{1}{4}\left(\Delta_{0} \phi\right) u, u\right\rangle .
\end{aligned}
$$

Proposition 2.1 follows from the various definitions

$$
\int_{\mathscr{D}}|\bar{\partial} u|^{2} \Phi=\|\bar{\partial} u\|^{2} \geq\left\langle-\frac{1}{4}\left(\Delta_{0} \phi\right), u, u\right\rangle=\int_{\mathscr{D}} \frac{1}{4}\left(\Delta_{0} \ln \Phi\right)|u|^{2} \Phi .
$$

We will specialize the choice of $\Phi$ to obtain some useful inequalities. In contrast to the most standard Carleman estimates, these inequalities will involve weight functions with singularities at several points. This feature of multiple singularities will be crucial in our eventual study of the nodal set for eigenfunctions.

Lemma 2.2. There exists a smooth function $\psi_{0}(z)$ defined for $|z|>1-2 a$ satisfying the following properties:

(i) $\psi_{0}(z)$ is bounded above and below by positive constants $c_{1} \leq \psi_{0}(z) \leq c_{2}$.

(ii) If $|z|>1$, then $\psi_{0}(z)=1$.

(iii) For all $z$ in the domain of $\psi_{0}, \Delta_{0} \ln \psi_{0} \geq 0$.

(iv) If $1-2 a<|z|<1-a$, then $\Delta_{0} \ln \psi_{0} \geq c_{3}>0$.

Proof. We choose $\psi_{0}(z)$ to be a radial function depending only on $r=|z|$. Let $h(r) \geq 0$ be a suitable smooth function satisfying $h(r) \geq c_{3}$ for $1-2 a<$ $|z|<1-a$, and $h(r)=0$ for $|z|>1-\frac{a}{2}$. The radial Laplacian

$$
\Delta_{0} \ln \psi_{0}(r)=\left(\frac{d^{2}}{d r^{2}}+\frac{1}{r} \frac{d}{d r}\right) \ln \psi_{0}(r)
$$

has smooth coefficients for $r>1-2 a$. Therefore, we may apply the existence and uniqueness theory for ordinary differential equations. Simply let $\ln \psi_{0}(r)$ be the solution of the differential equation $\left(\frac{d^{2}}{d r^{2}}+\frac{1}{r} \frac{d}{d r}\right) \ln \psi_{0}(r)=h(r)$ with initial conditions given by $\ln \psi_{0}(1)=0$ and $\ln \psi_{0}^{\prime}(1)=0$.

Next, let $D_{\nu}$ be a finite collection of pairwise disjoint disks, all of which are contained in the unit disk centered at the origin in $C$. We assume that $D_{\nu}=\left\{z|| z-z_{\nu} \mid<\delta\right\}$. Suppose that $D_{\nu}(a)$ denotes the smaller concentric disk $D_{\nu}(a)=\left\{z|| z-z_{\nu} \mid \leq(1-2 a) \delta\right\}$. We define a smooth weight function $\Phi_{0}(z)$ for $z \in C-\bigcup_{\nu} D_{\nu}(a)$ by setting $\Phi_{0}(z)=1$ when $z \notin \bigcup_{\nu} D_{\nu}$ and 
$\Phi_{0}(z)=\psi_{0}\left(\left(z-z_{\nu}\right) / \delta\right)$ when $z$ is an element of $D_{\nu}$. It follows from Lemma 2.2 that $\Phi_{0}$ satisfies the properties:

(i) $\Phi_{0}(z)$ is bounded above and below by positive constants $c_{1} \leq \Phi_{0}(z) \leq$ $c_{2}$.

(ii) $\Delta_{0} \ln \Phi_{0} \geq 0$ for all $z \in C-\bigcup_{\nu} D_{\nu}(a)$, the domain where the function $\Phi_{0}$ is defined.

(iii) $\Delta_{0} \ln \Phi_{0} \geq c_{3} \delta^{-2}$ when $(1-2 a) \delta<\left|z-z_{\nu}\right|<(1-a) \delta$.

Let $A_{\nu}$ denote the annulus $A_{\nu}=\left\{(1-2 a) \delta<\left|z-z_{\nu}\right|<(1-a) \delta\right\}$, and set $A=\bigcup_{\nu} A_{\nu}$. The properties (ii) and (iii) of $\Phi_{0}$ may be summarized as $\Delta_{0} \ln \Phi_{0} \geq c_{3} \delta^{-2} \chi_{A}$, where $\chi_{A}$ is the characteristic function of $A$.

Suppose that $\alpha$ is a nonnegative real constant. We apply Proposition 2.1 with $\Phi(z)=\Phi_{0}(z) e^{\alpha|z|^{2}}$. If $u \in C_{0}^{\infty}\left(R^{2}-\bigcup_{\nu} D_{\nu}(a)\right)$, assume that $\mathscr{D}$ is a bounded domain containing the support of $u$ and $A \subset \mathscr{D} \subset R^{2}-\bigcup_{\nu} D_{\nu}(a)$. A calculation gives

$$
\int_{\mathscr{D}}|\bar{\partial} u|^{2} \Phi_{0}(z) e^{\alpha|z|^{2}} \geq c_{4} \alpha \int_{\mathscr{D}}|u|^{2} \Phi_{0} e^{\alpha|z|^{2}}+c_{5} \delta^{-2} \int_{A}|u|^{2} \Phi_{0} e^{\alpha|z|^{2}} .
$$

The boundedness, property (i) of $\Phi_{0}$, then yields

$$
\int_{\mathscr{D}}|\bar{\partial} u|^{2} e^{\alpha|z|^{2}} \geq c_{6} \alpha \int_{\mathscr{D}}|u|^{2} e^{\alpha|z|^{2}}+c_{7} \delta^{-2} \int_{A}|u|^{2} e^{\alpha|z|^{2}} .
$$

We replace $u$ by $\frac{u}{P}$ in this inequality, where $P(z)=\prod_{\nu}\left(z-z_{\nu}\right)$. Since $P$ is holomorphic, we have $\bar{\partial}\left(\frac{u}{P}\right)=\frac{\bar{\partial} u}{P}$. Thus, we may write

Proposition 2.3. If $\mathscr{D}$ is a bounded domain containing $A$ and the support of $u$, then

$$
\int_{\mathscr{D}}|\bar{\partial} u|^{2}|P|^{-2} e^{\alpha|z|^{2}} \geq c_{6} \alpha \int_{\mathscr{D}}|u|^{2} e^{\alpha|z|^{2}}|P|^{-2}+c_{7} \delta^{-2} \int_{A}|u|^{2} e^{\alpha|z|^{2}}|P|^{-2} .
$$

The entire discussion above applies to any complex-valued smooth function $u$, with suitable compact support. Now let $f \in C_{0}^{\infty}\left(R^{2}-\bigcup_{\nu} \mathscr{D}_{\nu}(a)\right)$ be real valued. For real-valued functions, one has $|\bar{\partial} f|=|\partial f|=\frac{1}{2}|d f|$, where $d$ denotes the usual exterior derivative in $R^{2}$. We may choose $u=\partial f$.

Consequently, Proposition 2.3 yields

$$
\int_{\mathscr{D}}\left|\Delta_{0} f\right|^{2}|P|^{-2} e^{\alpha|z|^{2}} \geq c_{8} \alpha \int_{\mathscr{D}}|\bar{\partial} f|^{2} e^{\alpha|z|^{2}}|P|^{-2}+c_{9} \delta^{-2} \int_{A}|d f|^{2} e^{\alpha|z|^{2}}|P|^{-2} \text {. }
$$

Applying Proposition 2.1 to bound the first integral on the right-hand side gives

$$
\int_{\mathscr{D}}\left|\Delta_{0} f\right|^{2}|P|^{-2} e^{\alpha|z|^{2}} \geq c_{10} \alpha^{2} \int_{\mathscr{D}}|f|^{2} e^{\alpha|z|^{2}}|P|^{-2}+c_{9} \delta^{-2} \int_{A}|d f|^{2} e^{\alpha|z|^{2}}|P|^{-2}
$$

We would like to replace $d f$ by $f$ in the second integral on the right-hand side of (2.4). To achieve this goal, we impose the following hypotheses involving 
the geometry of the disks $D_{\nu}$ and the positive parameter $\alpha$ :

(i) The radius $\delta$ of each $D_{\nu}$ satisfies $\delta \leq b_{1} \alpha^{-1}$.

(ii) The distance between any two distinct $z_{\nu}$ is at least $2 b_{2} \max \left(\alpha^{1 / 2}, 1\right) \delta$.

(iii) The total number of disks $D_{\nu}$ is at most $b_{3} \alpha$.

Here $b_{i}$ are suitable positive constants. We may write

Lemma 2.6. Assume the hypotheses (2.5). If $\hat{z}_{1}$ and $\hat{z}_{2}$ both lie in the same component $A_{\nu}$ of $A$, then

(a) $b_{4}<e^{\alpha\left|\hat{z}_{1}\right|^{2}} / e^{\alpha\left|\hat{z}_{2}\right|^{2}}<b_{5}$.

(b) $b_{6}<\left|P\left(\hat{z}_{1}\right)\right| /\left|P\left(\hat{z}_{2}\right)\right|<b_{7}$.

Proof. To prove (a), recall that the $A_{\nu}$ all lie inside the unit disk, centered at the origin, in $R^{2}$. Thus, one has $\|\left.\hat{z}_{1}\right|^{2}-\left|\hat{z}_{2}\right|^{2}|\leq 2|\left|\hat{z}_{1}\right|-\left|\hat{z}_{2}\right| \mid \leq 2 b_{1} \alpha^{-1}$, by (i) of hypothesis (2.5). Therefore, $\left.\alpha|| \hat{z}_{1}\right|^{2}-\left|\hat{z}_{2}\right|^{2} \mid \leq 2 b_{1}$, and (a) follows via exponentiation.

For (b), we use the definition $P(z)=\prod_{\nu}\left(z-z_{\mu}\right)$, where $z_{\mu}$ are the distinct centers of the disks $D_{\mu}$. This gives

$$
\begin{aligned}
|\log | P\left(\hat{z}_{1}\right)|-\log | P\left(\hat{z}_{2}\right)|| & \leq \sum_{\mu}|\log | \hat{z}_{1}-z_{\mu}|-\log | \hat{z}_{2}-z_{\mu}|| \\
& \leq b_{8}+\sum_{\mu \neq \nu}|\log | \hat{z}_{1}-z_{\mu}|-\log | \hat{z}_{2}-z_{\mu}|| .
\end{aligned}
$$

Here $b_{8}=\log ((1-a) /(1-2 a))$, since $\hat{z}_{1}$ and $\hat{z}_{2}$ both lie in the annulus $A_{\nu}=\left\{z|(1-2 a) \delta<| z-z_{\nu} \mid<(1-a) \delta\right\}$.

By the mean value theorem, we get

$$
|\log | \hat{z}_{1}-z_{\mu}|-\log | \hat{z}_{2}-z_{\mu}|| \leq b_{9}\left|z_{\nu}-z_{\mu}\right|^{-1} \delta
$$

from hypothesis (ii) of (2.5). So

$$
|\log | P\left(\hat{z}_{1}\right)|-\log | P\left(\hat{z}_{2}\right)|| \leq b_{8}+b_{9} \delta \sum_{\mu \neq \nu}\left|z_{\nu}-z_{\mu}\right|^{-1} .
$$

To estimate the last sum, let $\bar{D}_{\mu}=\left\{z|| z-z_{\mu} \mid<b_{2} \max \left(\alpha^{1 / 2}, 1\right) \delta\right\}$. By assumption (ii) of (2.5), the $\bar{D}_{\mu}$ are disjoint. If $z \in \bar{D}_{\mu}$, then we have $\left|z-z_{\nu}\right| \leq$ $\left|z-z_{\mu}\right|+\left|z_{\mu}-z_{\nu}\right| \leq 2\left|z_{\mu}-z_{\nu}\right|$. Consequently,

$$
\int_{\bar{D}_{\mu}}\left|z-z_{\nu}\right|^{-1} \geq \frac{1}{2} \int_{\bar{D}_{\mu}}\left|z_{\mu}-z_{\nu}\right|^{-1} \geq b_{10}\left|z_{\mu}-z_{\nu}\right| \max (\alpha, 1) \delta^{2} .
$$

Here we use the definition of $\bar{D}_{\mu}$ to estimate its area.

Set $E_{\nu}=\bigcup_{\mu \neq \nu} \bar{D}_{\mu}$. We now have

$$
|\log | P\left(\hat{z}_{1}\right)|-\log | P\left(\hat{z}_{2}\right)|| \leq b_{8}+b_{11} \min \left(\alpha^{-1}, 1\right) \delta^{-1} \int_{E_{\nu}}\left|z-z_{\nu}\right|^{-1} .
$$


If $S$ is any set of finite measure $m(S)$, then let $D_{S}$ be a disk centered at $z_{\nu}$ with equal measure $m\left(D_{S}\right)=m(S)$. It follows from the elementary Lebesgue theory that

$$
\int_{S}\left|z-z_{\nu}\right|^{-1} \leq \int_{D_{S}}\left|z-z_{\nu}\right|^{-1}
$$

Applying this observation with $S=E_{\nu}$, we find

$$
|\log | P\left(\hat{z}_{1}\right)|-\log | P\left(\hat{z}_{2}\right)|| \leq b_{8}+b_{11} \min \left(\alpha^{-1}, 1\right) \delta^{-1} \int_{D_{E_{\nu}}}\left|z-z_{\nu}\right|^{-1} .
$$

By hypothesis (ii) of (2.5), we have $m\left(E_{\nu}\right) \leq b_{12} \alpha \max (\alpha, 1) \delta^{2}$. Since $E_{\nu}$ and $D_{E_{\nu}}$ have equal measure, part (b) of Lemma 2.6 follows easily.

We will also need a simple variant of the Poincare inequality on each annulus.

Lemma 2.7. If $f \in C^{\infty}\left(A_{\nu}\right)$ and $f$ vanishes on the inner boundary of $A_{\nu}$, then

$$
\int_{A_{\nu}}|d f|^{2} \geq c_{11} \delta^{-2} \int_{A_{\nu}}|f|^{2}
$$

Proof. Let $(r, \theta)$ denote the usual polar coordinates on $A_{\nu}$. Since $f$ vanishes on $r=(1-2 a) \delta$, the fundamental theorem of calculus yields $f(r, \theta)=$ $\int_{(1-2 a) \delta}^{r} \partial f / \partial s(s, \theta) d s$. Thus,

$$
\int_{A_{\nu}}|f|^{2}=\int_{0}^{2 \pi} \int_{(1-2 a) \delta}^{(1-a) \delta}\left(\int_{(1-2 a) \delta}^{r} \frac{\partial f}{\partial s}(s, \theta) d s\right)^{2} r d r d \theta .
$$

By the Schwarz inequality,

$$
\begin{aligned}
\int_{A_{\nu}}|f|^{2} & \leq \int_{0}^{2 \pi} \int_{(1-2 a) \delta}^{(1-a) \delta} a \delta \int_{(1-2 a) \delta}^{(1-a) \delta}\left(\frac{\partial f}{\partial s}\right)^{2}(s, \theta) d s r d r d \theta \\
& \leq c_{12} \delta^{2} \int_{0}^{2 \pi} \int_{(1-2 a) \delta}^{(1-a) \delta}\left(\frac{\partial f}{\partial s}\right)^{2}(s, \theta) s d s d \theta .
\end{aligned}
$$

Since $|d f|^{2}(s, \theta) \geq\left(\frac{\partial f}{\partial s}\right)^{2}(s, \theta)$, Lemma 2.7 follows.

Finally, we are ready to return to (2.4). Let $w_{\nu} \in A_{\nu}$ be chosen arbitrarily. Assume the hypotheses (2.5). Then applying Lemma 2.6,

$$
\int_{A}|d f|^{2} e^{\alpha|z|^{2}}|P(z)|^{-2} \geq c_{13} \sum_{\nu} e^{\alpha\left|w_{\nu}\right|^{2}}\left|P\left(w_{\nu}\right)\right|^{-2} \int_{A_{\nu}}|d f|^{2} .
$$

Since $f \in C_{0}^{\infty}\left(R^{2}-\bigcup_{\nu} D_{\nu}(a)\right)$, we then employ Lemma 2.7 to obtain

$$
\int_{A}|d f|^{2} e^{\alpha|z|^{2}}|P(z)|^{-2} \geq c_{14} \sum_{\nu} e^{\alpha\left|w_{\nu}\right|^{2}}\left|P\left(w_{\nu}\right)\right|^{-2} \delta^{-2} \int_{A_{\nu}}|f|^{2} .
$$

Using Lemma 2.6 again gives

$$
\int_{A}|d f|^{2} e^{\alpha|z|^{2}}|P(z)|^{-2} \geq c_{15} \delta^{-2} \int_{A}|f|^{2} e^{\alpha|z|^{2}}|P(z)|^{-2} .
$$


We obtain a crucial Carleman inequality by substituting (2.9) back into (2.4),

$$
\begin{aligned}
\int_{\mathscr{D}}\left|\Delta_{0} f\right|^{2}|P|^{-2} e^{\alpha|z|^{2}} \geq & c_{10} \alpha^{2} \int_{\mathscr{D}}|f|^{2} e^{\alpha|z|^{2}}|P|^{-2} \\
& +c_{16} \delta^{-4} \int_{A}|f|^{2} e^{\alpha|z|^{2}}|P|^{-2} .
\end{aligned}
$$

For future reference, it is convenient to summarize the principal results from this section in

Proposition 2.11. Assume that $f \in C_{0}^{\infty}\left(R^{2}-\bigcup_{\nu} D_{\nu}(a)\right)$ is a real-valued function. Let $\mathscr{D}$ be a bounded domain containing the support of $f$. Then

(i) for any disk $D_{\nu}$,

$$
\int_{\mathscr{D}}\left|\Delta_{0} f\right|^{2}|P|^{-2} e^{\alpha|z|^{2}} \geq c_{10} \alpha^{2} \int_{\mathscr{D}}|f|^{2} e^{\alpha|z|^{2}}|P|^{-2} \text {. }
$$

(ii) If the $D_{\nu}$ satisfy hypotheses (2.5), then the stronger inequality (2.10) holds.

Note that the estimate of Proposition 2.11(i) is a consequence of (2.4), which was proved without assuming special hypotheses about the disks $D_{\nu}$. In fact, (i) does not even require the $D_{\nu}$ to be disjoint. This follows from the disjoint case by a simple limiting argument. The point is that the radius of $D_{\nu}$ does not enter into the weak inequality of (i). Alternatively, we may deduce Proposition 2.11(i) directly from Proposition 2.1 by using the weight function $|P(z)|^{-2} \exp \left(\alpha|z|^{2}\right)$.

\section{COUNTING SINGULAR POINTS}

Let $M$ be a compact connected closed Riemannian manifold with associated Laplacian $\Delta$. Suppose that $F$ is an eigenfunction of $\Delta$ with eigenvalue $-\lambda$, $\Delta F=-\lambda F$. In [5], we proved that $F$ vanishes at any point $p$ in $M$ to at most order $c_{1} \sqrt{\lambda}$. The purpose of the present section is to make a qualitative improvement in this result assuming that $M$ is two dimensional.

Suppose that $M$ is a surface. The nodal set of $F$ is defined as $N=$ $\{x \in M \mid F(x)=0\}$. The implicit function theorem guarantees that $N$ is locally a one-dimensional manifold, outside its singular set $S=\{x \in M \mid F(x)=0$ and $d F(x)=0\}$. We begin by recalling the elementary

Lemma 3.1. $S$ consists of at most finitely many points.

Proof. By the unique continuation theory [1], $F$ never vanishes to infinite order. Let $p_{0} \in S$ and choose normal coordinates $(x, y)$ with $p_{0}$ at the origin. Expanding $F$ in its local Taylor polynomial, we have $F(x, y)=$ $F_{j}(x, y)+R_{j+1}(x, y)$. Here $F_{j}(x, y)$ consists of the leading nonvanishing terms, all those homogeneous of order $j \geq 2$, and $R_{j+1}(x, y)$ is a higher order remainder. Since the coordinates are normal and $\Delta F=-\lambda F$, we deduce that $F_{j}$ is harmonic for the Euclidean Laplacian $\Delta_{0} F_{j}=0$. Passing to polar coordinates, we find that $F_{j}=r^{j}\left(b_{1} \cos j \theta+b_{2} \sin j \theta\right)$. Clearly, $r^{-1} \partial F_{j} / \partial \theta$ and $\partial F_{j} / \partial r$ have no common zero for $r \neq 0$. Thus, $p_{0}$ has a neighborhood $U$ with $U \cap S=p_{0}$. Lemma 3.1 follows since $M$ is compact. 
We plan to count the number of singular points in sufficiently small balls. Let $p \in M$, and consider a geodesic ball $B\left(p, c_{2} \lambda^{-1 / 4}\right)$ of radius $c_{2} \lambda^{-1 / 4}$ and centered at $p$. If $c_{2}$ is sufficiently small, we may assume that $B\left(p, c_{2} \lambda^{-1 / 4}\right)$ is contained in an isothermal coordinate patch. Then we may write $\Delta=\phi_{1}^{-1} \Delta_{0}$ and $\Delta_{0} F=-\lambda \phi_{1} F$. Suppose we define $G(x)=F\left(c_{3} \lambda^{-1 / 4} x\right)$. If $c_{3}$ is suffciently small, the domain of $G$ will contain a Euclidean ball of radius three centered at the origin. Moreover, $\Delta_{0} G=-\lambda^{1 / 2} \phi_{2} G$, where we may make $\phi_{2}$ arbitrarily small by proper choice of $c_{3}$. With these preparations we are now ready to prove

Proposition 3.2. Suppose that $p_{\nu} \in S \cap B\left(p, c_{4} \lambda^{-1 / 4}\right)$ are singular points where $F$ vanishes to order $n_{\nu}+1$. Then $\sum_{\nu} n_{\nu}<c_{5} \lambda^{1 / 2}$.

Proof. If suffices to bound the number of singular points of $G$ that are contained in a Euclidean ball of radius $\frac{1}{10}$ centered at the origin. Suppose that $\left|z_{\nu}\right|<\frac{1}{10}$ and $G$ vanishes at $z_{\nu}$ to order $n_{\nu}+1$.

The singular points of order two require a special argument. Therefore, we begin by assuming $n_{\nu}=m_{\nu}+1$, where $m_{\nu} \geq 1$. We may then define the polynomial $P(z)=\Pi\left(z-z_{\nu}\right)^{m_{\nu}}$. Let $D$ be an open disk of radius two centered at the origin and $D_{\nu}$ closed small disjoint disks of radius $\delta$ centered at the $z_{\nu}$. If $f \in C_{0}^{\infty}\left(D-\bigcup_{\nu} D_{\nu}\right)$, the Proposition 2.11(i) gives

$$
\int_{D}\left|\Delta_{0} f\right|^{2}|P|^{-2} e^{b_{3} \sqrt{\lambda}|z|^{2}} \geq c_{6} \lambda \int_{D}|f|^{2}|P|^{-2} e^{b_{3} \sqrt{\lambda}|z|^{2}} .
$$

Here we choose $\alpha=b_{3} \sqrt{\lambda}$.

Of course, the function $G$ will not be compactly supported. Consequently, we employ a standard cut-off function $\theta \in C_{0}^{\infty}\left(D-\bigcup_{\nu} D_{\nu}\right)$ having properties

(i) $\theta(z)=1$ if $|z|<\frac{3}{2}$ and all $\left|z-z_{\nu}\right|>2 \delta$.

(ii) $|\nabla \theta| \leq c_{7},\left|\Delta_{0} \theta\right| \leq c_{8}$ if $|z| \geq \frac{3}{2}$.

(iii) $|\nabla \theta| \leq c_{7} \delta^{-1},\left|\Delta_{0} \theta\right| \leq c_{8} \delta^{-2}$ if $\left|z-z_{\nu}\right| \leq 2 \delta$.

We may take $f=\theta G$ in (3.3).

It is straightforward to derive the estimates

(i) $\left|\Delta_{0}(\theta G)\right| \leq c_{9} \delta^{m_{\nu}}$ if $\left|z-z_{\nu}\right| \leq 2 \delta$.

(ii) $\left|\Delta_{0}(\theta G)\right| \leq c_{10}(|G|+|\nabla G|+\sqrt{\lambda}|G|)$ if $|z| \geq \frac{3}{2}$.

In particular, $|P|^{-1} \Delta_{0}(\theta G) \mid$ is bounded near the zeros $z_{\nu}$ of $P$. The constant $c_{9}$ may depend upon $G$, but $c_{9}$ will soon disappear from our argument.

Substituting $f=\theta G$ in (3.3) and applying the dominated convergence theorem as $\delta \rightarrow 0$ gives

$$
\begin{aligned}
& \int_{|z|<3 / 2}\left|\Delta_{0} G\right|^{2}|P|^{-2} e^{b_{3} \sqrt{\lambda}|z|^{2}}+c_{11}(1+\lambda) \int_{3 / 2 \leq|z| \leq 2}\left(|G|^{2}+|\nabla G|^{2}\right)|P|^{-2} e^{b_{3} \sqrt{\lambda}|z|} \\
& \quad \geq c_{6} \lambda \int_{|z|<3 / 2}|G|^{2}|P|^{-2} e^{b_{3} \sqrt{\lambda}|z|^{2}} .
\end{aligned}
$$


Now $\Delta_{0} G=-\lambda^{1 / 2} \phi_{2} G$, where $\phi_{2}$ can be assumed suitably small. This allows the absorption of the first term on the left into the right-hand side. We find that

$$
\int_{3 / 2 \leq|z| \leq 2}\left(|G|^{2}+|\nabla G|^{2}\right)|P|^{-2} e^{b_{3} \sqrt{\lambda}|z|^{2}} \geq c_{12} \int_{|z| \leq 1 / 2}|G|^{2}|P|^{-2} e^{b_{3} \sqrt{\lambda}|z|^{2}} .
$$

Replacing $|P|^{-2}$ by its maximum or minimum value in each integral gives

$$
\begin{gathered}
\max _{|z| \geq 3 / 2}|P|^{-2}\left(\int_{3 / 2 \leq|z| \leq 2}|G|^{2}+|\nabla G|^{2}\right) e^{4 b_{3} \sqrt{\lambda}} \\
\geq c_{12}\left(\min _{|z| \leq 1 / 2}|P|^{-2}\right) \int_{|z|<1 / 2}|G|^{2} .
\end{gathered}
$$

By standard elliptic theory, the above implies

$$
\max _{|z| \geq 3 / 2}|P|^{-2} e^{b_{4} \sqrt{\lambda}} \int_{|z| \leq 5 / 2}|G|^{2} \geq c_{12} \min _{|z| \leq 1 / 2}|P|^{-2} \int_{|z| \leq 1 / 2}|G|^{2} .
$$

The basic estimate of [5] for growth of eigenfunctions on concentric balls now yields

$$
\begin{aligned}
e^{b_{5} \sum m_{\nu}} & \leq \frac{\min _{|z| \leq 1 / 2}|P|^{-2}}{\max _{|z| \geq 3 / 2}|P|^{-2}} \\
& \leq \frac{e^{b_{4} \sqrt{\lambda}} \int_{|z| \leq 5 / 2}|G|^{2}}{c_{12} \int_{|z| \leq 1 / 2}|G|^{2}} \\
& \leq e^{b_{6} \sqrt{\lambda}} .
\end{aligned}
$$

Since $m_{\nu} \geq 1$, we have $n_{\nu}=m_{\nu}+1 \leq 2 m_{\nu}$. We have thus proved Proposition 3.2 in the case where all singular points are of order at least three.

To treat the singular points of order two, we want to replace $P(z)$ by $Q(z)=$ $\Pi\left(z-z_{\nu}\right)^{n_{\nu} / 2}$ in the above argument. The point is that $|Q|^{-2}\left|\Delta_{0}(\theta G)\right|^{2}$ will then be uniformly integrable as $\delta \downarrow 0$ near the singular points $z_{\nu}$. Unfortunately, $Q$ need not be defined as a single-valued holomorphic function on $C$. To overcome this difficulty, we pass to a finite branched cover of the disk $D$ punctured at $z_{\nu}$. The Carleman inequalities of $\S 2$, which follow primarily by partial integration, generalize in a straightforward manner. The integrand in these inequalities will involve only functions such as $f$ and $|Q|$ that are independent of the sheet. So we do in fact have the required Carleman inequality on a punctured disk. Note that the points $z_{\nu}$ have Euclidean measure zero. Proposition 3.2 follows as in the case where all $n_{\nu}>1$.

We proceed to discuss some corollaries and extensions of Proposition 3.2. An elementary argument using Courant's nodal domain theorem and the GaussBonnet theorem implies that the cardinality of $S$ is at most of order $\lambda$. We omit the details since this result is also a consequence of Proposition 3.2. We 
simply cover $M$ by order $\lambda^{1 / 2}$ balls of radius $c_{4} \lambda^{-1 / 4}$ to deduce

Corollary 3.4. Suppose that $p_{\nu} \in S$ are singular points where $F$ vanishes to order $n_{\nu}+1$. Then $\sum n_{\nu} \leq c_{13} \lambda$.

Proposition 3.2 and its Corollary 3.4 are sharp in several respects. One has spherical harmonics on $S^{2}$ that vanish at a single point to order $\sqrt{\lambda}$. Moreover, let $T^{2}=R^{2} / Z \oplus Z$ be the standard flat two torus. If $F_{1} \in C^{\infty}\left(T^{2}\right)$ is an eigenfunction with eigenvalue $\lambda_{1}$, then set $F_{n}(x, y)=F_{1}(n x, n y)$. Clearly, $F_{n}$ is an eigenfunction with eigenvalue $\lambda_{n}=n^{2} \lambda_{1}$. If $F_{1}$ has a zero of order $m$, then $F_{n}$ has at least $b_{7} \lambda_{n}$ zeros of order $m$. This shows that the global Corollary 3.4 is sharp. Also, $B\left(p, c_{4} \lambda^{-1 / 4}\right)$ in Proposition 3.2 cannot be replaced by any $B\left(p, c_{14} \lambda^{-\varepsilon}\right)$ for $\varepsilon<\frac{1}{4}$ while still achieving at most order $\sqrt{\lambda}$ singularities in $B\left(p, c_{14} \lambda^{-\varepsilon}\right)$. If so, $F$ would have at most order $\lambda^{1 / 2+2 \varepsilon}$ singularities on all of $T^{2}$.

It may be interesting to discuss the geometric dependence of the constants appearing in our present results. We have

Proposition 3.5. The constants $c_{4}^{-1}$ and $c_{5}$ of Proposition 3.2 may be bounded above via an upper bound on the diameter and absolute value of the Gaussian curvature of $M$. If there is a lower bound for the value of $M$, then the constant $c_{13}$ of Corollary 3.4 can be bounded from above.

In fact, the eigenfunction growth estimate of [5] already required an upper bound for the diameter and absolute value of the curvature of $M$. To see that no additional geometric data is required for Proposition 3.2, we lift the metric to the tangent space at $p \in M$ before doing our basic calculation. This avoids the presence of cut points. Appealing to the Riemann mapping theorem of [9], we obtain a conformal coordinate chart on a ball in $T_{p} M$ where the radius of the ball and isothermal parameter are controlled. If we also have a lower bound for the volume of $M$, then Cheeger [4] provides a lower bound for the injectivity radius of $M$. This allows us to bound the number of balls with radius $c_{4} \lambda^{-1 / 4}$ needed to cover $M$. The constant $c_{13}$ of Corollary 3.4 is thereby controlled. Note that an upper bound for the diameter and lower bound for the Gaussian curvature already provide an upper bound for the volume of $M$. Using the Courant nodal domain theorem, as alluded to above, to prove Corollary 3.4, and lower bounds for the eigenvalues, we can improve the geometric dependence in Corollary 3.4. More specifically, the absolute bound on the curvature can be replaced by a lower bound on the curvature.

If $M$ is a manifold with boundary, then it is natural to impose Dirichlet or Neumann boundary conditions on the eigenfunction $F$. Proposition 3.2 and Corollary 3.4 generalize in a straightforward way. As in [6], we work on the Lipschitz double of $M$. The only delicate point is to construct suitable conformal coordinate charts near $\partial M \subset M \cup M$. For this, we apply the Riemann mapping theorem of [9] to first construct charts around $\partial M \subset M$. We map 
a half disk centered on the $x$-axis in the $(x, y)$-plane to $M$ with the $x$-axis going to $\partial M$. The metric is then given locally by $h(x, y)\left(d x^{2}+d y^{2}\right)$ with $y>0$. The definition of the metric and differentiable structure on the double correspond to reflection about the $x$-axis. This gives the required charts with metric $h(x,|y|)\left(d x^{2}+d y^{2}\right)$ on the double $M \cup M$.

This section includes the completion of the proof for Theorem 1.1. In fact, Theorem 1.1 is contained in Proposition 3.2 and the subsequent developments.

\section{GROWTH OF EIGENFUNCTIONS}

The remainder of this manuscript is devoted to the proof for Theorem 1.2. It requires considerable effort to develop the necessary machinery. The present section establishes that our eigenfunction does not grow rapidly on too many small balls. These growth estimates will subsequently be shown to restrict the nodal length.

Let $M$ be a compact closed Riemannian surface. Assume that $\Delta F=-\lambda F$, where $\Delta$ is the Laplacian of the Riemannian metric. We consider the behavior of $F$ when restricted to small balls $B\left(p, c_{1} \lambda^{-1 / 4}\right)$ contained in isothermal coordinate patches. We may write $\Delta=\phi_{1}^{-1} \Delta_{0}$, where $\Delta_{0}$ is the Euclidean Laplace operator. Consequently, we have $\Delta_{0} F=-\lambda \phi_{1} F$.

Define $G(z)=F\left(c_{2} \lambda^{-1 / 4} z\right)$. If $c_{2}$ is sufficiently small, then $G$ will be well defined on a Euclidean ball of radius three centered at the origin of $R^{2}$. Moreover, $\Delta_{0} G=-\lambda^{1 / 2} \phi_{2} G$, where we may assume that $\phi_{2}$ is arbitrarily small. Our intention is to apply the Carleman estimate (2.10) with $f=\theta G$, where $\theta$ is a suitable cut-off function.

To use Proposition 2.11(ii), we need a collection $D_{\nu}=\left\{z|| z-z_{\nu} \mid<\delta\right\}$ of disjoint disks satisfying hypotheses (2.5). Here we assume that $\alpha=b_{1} \sqrt{\lambda}$. Moreover, we will require that all $D_{\nu}$ lie in a ball of radius $\frac{1}{30}$ centered at the origin in $R^{2}$. Following the notation of $\S 2$, we define $D_{\nu}(a)=\left\{z|| z-z_{\nu} \mid \leq\right.$ $(1-2 a) \delta\}$, where $a$ is a suitably small positive constant.

If $D$ is an open Euclidean disk with radius two centered at the origin, let $\theta \in C_{0}^{\infty}\left(D-\bigcup_{\nu} D_{\nu}(a)\right)$. Furthermore, assume that $\theta$ satisfies

(i) $\theta(z)=1$ if $|z|<1$ and $\left|z-z_{\nu}\right|>\left(1-\frac{3}{2} a\right) \delta$ for all $\nu$.

(ii) $\left|\Delta_{0} \theta\right|+|d \theta|<c_{3}$ if $|z| \geq 1$.

(iii) $\left|\Delta_{0} \theta\right| \leq c_{4} \delta^{-2},|d \theta| \leq c_{5} \delta^{-1}$ if $\left|z-z_{\nu}\right|<\delta\left(1-\frac{3}{2} \alpha\right)$.

It is elementary to construct such cut-off functions $\theta$.

Substituting $f=\theta g$ in (2.10) yields

$$
\begin{aligned}
\int_{D}\left|\Delta_{0}(\theta G)\right|^{2}|P|^{-2} e^{b_{1} \sqrt{\lambda}|z|^{2}} \\
\geq c_{6} \lambda \int_{D} \theta^{2} G^{2} e^{b_{1} \sqrt{\lambda}|z|^{2}}|P|^{-2} \\
\quad+c_{7} \delta^{-4} \int_{A} \theta^{2} G^{2} e^{b_{1} \sqrt{\lambda}|z|^{2}}|P|^{-2} .
\end{aligned}
$$

Here $A=\bigcup_{\nu} A_{\nu}$ and $A_{\nu}=\left\{z|(1-2 a) \delta<| z-z_{\nu} \mid<(1-a) \delta\right\}$ is an annulus. 
Consider the integral on the left-hand side. We have

$$
\left|\Delta_{0}(\theta G)\right|^{2}=\left|\theta \Delta_{0} G+2\langle\nabla \theta, \nabla G\rangle+G \Delta_{0} \theta\right|^{2} .
$$

Using the eigenvalue equation $\Delta_{0} G=-\lambda^{1 / 2} \phi_{2} G$, we deduce a basic inequality. It is

$$
\left|\Delta_{0}(\theta G)\right|^{2} \leq b_{2}\left(\theta^{2} \lambda \phi_{2}^{2} G^{2}+|\nabla \theta|^{2}|\nabla G|^{2}+G^{2}\left|\Delta_{0} \theta\right|^{2}\right) .
$$

If $\phi_{2}$ is sufficiently small, we may absorb the integral term involving $\theta^{2} G^{2}$ into the right-hand side. This gives

$$
\begin{aligned}
& \int_{D}\left(|\nabla \theta|^{2}|\nabla G|^{2}+G^{2}\left|\Delta_{0} \theta\right|^{2}\right)|P|^{-2} e^{b_{1} \sqrt{\lambda}|z|^{2}} \\
& \quad \geq c_{8} \lambda \int \theta^{2} G^{2} e^{b_{1} \sqrt{\lambda}|z|^{2}}|P|^{-2}+c_{7} \delta^{-4} \int_{A} \theta^{2} G^{2} e^{b_{1} \sqrt{\lambda}|z|^{2}}|P|^{-2} .
\end{aligned}
$$

We now bound the right-hand side using property (i) of $\theta$. We obtain

$$
\begin{aligned}
& \int_{D}\left(|\nabla \theta|^{2}|\nabla G|^{2}+G^{2}\left|\Delta_{0} \theta\right|^{2}|P|^{-2} e^{b_{1} \sqrt{\lambda}|z|^{2}}\right. \\
& \geq c_{8} \lambda \int_{1 / 4<|z|<1 / 2} G^{2} e^{b_{1} \sqrt{\lambda}|z|^{2}}|P|^{-2} \\
& \quad+c_{7} \delta^{-4} \sum_{\nu} \int_{(1-3 a / 2) \delta<\left|z-z_{\nu}\right|<(1-a) \delta} G^{2} e^{b_{1} \sqrt{\lambda}|z|^{2}}|P|^{-2} .
\end{aligned}
$$

Here the assumption that all $D_{\nu}$ lie in a ball of radius $\frac{1}{30}$ centered at the origin was employed.

The definition of $\theta$ shows that we may decompose the left-hand side of (4.1) as a sum $I+\sum_{\nu} I_{\nu}$ corresponding to the outer part of $D$ and the $A_{\nu}$. More precisely,

$$
I=\int_{1<|z|<2}\left(|\nabla \theta|^{2}|\nabla G|^{2}+G^{2}\left|\Delta_{0} \theta\right|^{2}\right)|P|^{-2} e^{b_{1} \sqrt{\lambda}|z|^{2}} .
$$

Applying standard elliptic theory gives

$$
I \leq e^{b_{3} \sqrt{\lambda}}\left(\int_{3 / 4<|z|<5 / 2}|G|^{2}\right) \max _{|z|>1}|P|^{-2} .
$$

Similarly, we have

$$
I_{\nu}=\int_{(1-2 a) \delta<\left|z-z_{\nu}\right|<(1-3 a / 2) \delta}\left(|\nabla \theta|^{2}|\nabla G|^{2}+G^{2}\left|\Delta_{0} \theta\right|^{2}\right)|P|^{-2} e^{b_{1} \sqrt{\lambda}|z|^{2}}
$$

and via elliptic theory

$$
I_{\nu} \leq c_{9}\left(\int_{(1-3 a) \delta<\left|z-z_{\nu}\right|<(1-4 a / 3) \delta} \delta^{-4}|G|^{-2}\right) \max _{A_{\nu}}\left(|P|^{-2} e^{b_{1} \sqrt{\lambda}|z|^{2}}\right) .
$$

Appealing to Lemma 2.6 gives

$$
I_{\nu} \leq c_{10} \delta^{-4}\left(\int_{(1-3 a) \delta<\left|z-z_{\nu}\right|<(1-4 a / 3) \delta}|G|^{2}\right) \min _{A_{\nu}}\left(|P|^{-2} e^{b_{1} \sqrt{\lambda}|z|^{2}}\right) .
$$


Substituting our upper bounds (4.2) and (4.3) back into (4.1) yields

$$
\begin{aligned}
& \int_{3 / 4<|z|<5 / 2}|G|^{2} e^{b_{3} \sqrt{\lambda}} \max _{|z|<1}|P|^{-2} \\
&+c_{10} \delta^{-4} \sum_{\nu}\left(\int_{(1-3 a) \delta<\left|z-z_{\nu}\right|<(1-4 a / 3) \delta}|G|^{2}\right) \min _{A_{\nu}}\left(|P|^{-2} e^{b_{1} \sqrt{\lambda}|z|^{2}}\right) \\
& \geq c_{11}\left(\int_{1 / 4<|z|<1 / 2}|G|^{2}\right) \min _{|z|<1 / 2}|P|^{-2} \\
&+c_{12} \delta^{-4} \sum_{\nu}\left(\int_{(1-3 a / 2) \delta<\left|z-z_{\nu}\right|<(1-a) \delta}|G|^{2}\right) \min _{A_{\nu}}\left(|P|^{-2} e^{b_{1} \sqrt{\lambda}|z|^{2}}\right) .
\end{aligned}
$$

The growth estimate of [5] for eigenfunctions implies that if the number of disks $D_{\nu}$ is $b_{6} \sqrt{\lambda}$, then

$$
\int_{3 / 4<|z|<5 / 2}|G|^{2} e^{b_{3} \sqrt{\lambda}} \max _{|z|>1}|P|^{-2}<c_{11} \int_{1 / 4<|z|<1 / 2}|G|^{2} \min _{|z|<1 / 2}|P|^{-2} .
$$

The constant $b_{6}$ here is proportional to the constant $b_{3}$ in (2.5)(iii). To apply [5], we must choose this constant sufficiently large depending on the curvature and diameter of $M$. This will not affect the argument of $\S 2$.

Suppose that in addition we have for all $\nu$

$$
c_{13} \int_{(1-3 a) \delta<\left|z-z_{\nu}\right|<(1-4 a / 3) \delta}|G|^{2} \leq \int_{(1-3 a / 2) \delta<\left|z-z_{\nu}\right|<(1-a) \delta}|G|^{2} .
$$

Clearly, the last supposition (4.6) is incompatible with (4.4) and (4.5). Therefore, the above hypotheses cannot all be valid.

It is useful to reformulate and summarize our conclusion for future reference. We do this in

Proposition 4.7. Let $G(z)=F\left(c_{2} \lambda^{-1 / 4} z\right)$ be defined on a Euclidean ball of radius three centered at the origin. Suppose that $D_{\nu}=\left\{z|| z-z_{\nu} \mid<\delta\right\}$ are disks contained in a Euclidean ball of radius $\frac{1}{30}$ centered at 0 . In addition assume that (i) $\delta<b_{7} \lambda^{-1 / 2}$ and (ii) $\left|z_{\mu}-z_{\nu}\right|>b_{8} \lambda^{1 / 4} \delta$, when $\mu \neq \nu$. If (4.6) holds for all $\nu$, then the number of disks $D_{\nu}$ is less than $b_{6} \sqrt{\lambda}$.

\section{NODAL LENGTH AND GROWTH ESTIMATES}

The present section is devoted to a local study of the relationship between growth of eigenfunctions and nodal length. We demonstrate that a suitable upper bound for the growth in $L^{2}$ norm on given balls implies an upper bound for the nodal length on smaller concentric balls. It appears that one could alternatively rely upon the work of Hardt and Simon [8] for the principal results of this section. However, we prefer to pursue an approach that is more in the spirit of our earlier work.

Everything will be developed in the framework of balls centered at the origin 0 in $R^{2}$. The Euclidean distance from 0 is denoted by $r$, and $\Delta_{0}$ denotes the 
Euclidean Laplacian. Suppose that $\phi$ is a sufficiently small smooth function and $\bar{\varepsilon}_{0}, \varepsilon_{0}$ are suitably small positive real numbers. The quantitative Aronszajn estimate of [5] specializes to give

Lemma 5.1. Let $u \in C_{0}^{\infty}\left(\frac{1}{2} \bar{\varepsilon}_{0}<r<\varepsilon_{0}\right)$. If $\beta>b_{1}$, then

$$
\int \bar{r}^{2(2-\beta)}\left|\left(\Delta_{0}+\phi\right) u\right|^{2} r^{-1} d r d w \geq c_{1} \beta^{2} \int \bar{r}^{2-2 \beta} u^{2} r^{-1} d r d w .
$$

Here $\bar{r}$ is the geodesic distance of a metric commensurable with the Euclidean metric. Moreover, $(r, w)$ are the standard polar coordinates.

Let $H$ be a solution of the partial differential equation $\Delta_{0} H=-\phi H$ valid at all points in the unit ball in $R^{2}$. The reader may anticipate that we plan to substitute $u=\theta H$ in Lemma 5.1, where $\theta$ is a suitable cut-off function. We will use the ensuing calculation to prove

Lemma 5.2. Suppose that $H$ satisfies the growth estimate

$$
\int_{(1-3 a / 2) \varepsilon_{0}<r<(1-a) \varepsilon_{0}} H^{2} \leq c_{2} \int_{(1-3 a) \varepsilon_{0}<r<(1-4 a / 3) \varepsilon_{0}} H^{2},
$$

where $a$ is a fixed small constant. Then for $0<\bar{\varepsilon}_{0}<\varepsilon_{0} / 100$, we have

$$
\max _{r \leq \bar{\varepsilon}_{0}}|H|^{2} \geq c_{3}\left(\frac{\bar{\varepsilon}_{0}}{\varepsilon_{0}}\right)^{2 c_{4}} \underset{r<(1-4 a / 3) \varepsilon_{0}}{\operatorname{Av}}|H|^{2} .
$$

Here Av denotes the average value of $|H|^{2}$ on the set $r<(1-4 a / 3) \varepsilon_{0}$.

Proof. We construct a radial cut-off function $\theta \in C_{0}^{\infty}\left(\frac{1}{2} \bar{\varepsilon}_{0}<r<\left(1-\frac{11}{10} a\right) \varepsilon_{0}\right)$ satisfying

(i) $\theta(r)=1$ for $\frac{3}{4} \bar{\varepsilon}_{0}<r<\left(1-\frac{10}{9} a\right) \varepsilon_{0}$.

(ii) $|d \theta|+\left|\Delta_{0} \theta\right| \leq c_{5}$ for $r>\left(1-\frac{10}{9} a\right) \varepsilon_{0}$.

(iii) $|d \theta| \leq c_{6} \bar{\varepsilon}_{0}^{-1},\left|\Delta_{0} \theta\right| \leq c_{7} \bar{\varepsilon}_{0}^{-2}$ for $r \leq \frac{3}{4} \bar{\varepsilon}_{0}$.

Clearly, $\left(\Delta_{0}+\phi\right)(\theta H)=\Delta_{0} \theta H+2\langle\nabla \theta, \nabla H\rangle$.

Setting $u=\theta H$ in Lemma 5.1 yields

$$
I \geq c_{1} \beta^{2} \int \bar{r}^{2-2 \beta} \theta^{2} H^{2} r^{-1} d r d w,
$$

where

$$
I=\int \bar{r}^{2(2-\beta)}\left|\Delta_{0} \theta H+2\langle\nabla \theta, \nabla H\rangle\right|^{2} r^{-1} d r d w .
$$

Clearly, since $\bar{r}=r+O\left(r^{2}\right)$,

$$
\begin{aligned}
I \leq & \left(\frac{1}{4} \bar{\varepsilon}_{0}\right)^{2(2-\beta)} \int_{\bar{\varepsilon}_{0} / 2<r<3 \bar{\varepsilon}_{0} / 4}\left|\Delta_{0} \theta H+2\langle\nabla \theta, \nabla H\rangle\right|^{2} r^{-2} d r d w \\
& +c_{8}\left[\left(1-\frac{11}{9} a\right) \varepsilon_{0}\right]^{-2 \beta} \\
& \times \int_{(1-10 a / 9) \varepsilon_{0}<r<(1-11 a / 10) \varepsilon_{0}}\left|\Delta_{0} \theta H+2\langle\nabla \theta, \nabla H\rangle\right|^{2} r^{-1} d r d w .
\end{aligned}
$$


Applying standard elliptic theory, we deduce

$$
\begin{aligned}
I \leq & c_{9}\left(\frac{1}{4} \bar{\varepsilon}_{0}\right)^{-2 \beta} \int_{\bar{\varepsilon}_{0} / 4<r<\bar{\varepsilon}_{0}} H^{2} r^{-1} d r d w \\
& +c_{10}\left[\left(1-\frac{11}{9} a\right) \varepsilon_{0}\right]^{-2 \beta} \int_{(1-3 a / 2) \varepsilon_{0}<r<(1-a) \varepsilon_{0}} H^{2} r^{-1} d r d w .
\end{aligned}
$$

Returning to (5.3), we may write

$$
\begin{aligned}
{\left[\left(1-\frac{11}{9} a\right) \varepsilon_{0}\right]^{-2 \beta} c_{10} \int_{(1-3 a / 2) \varepsilon_{0}<r<(1-a) \varepsilon_{0}} H^{2} r^{-1} d r d w } \\
\quad+c_{9}\left(\frac{1}{4} \bar{\varepsilon}_{0}\right)^{-2 \beta} \int_{\bar{\varepsilon}_{0} / 4<r<\bar{\varepsilon}_{0}} H^{2} r^{-1} d r d w \\
\geq c_{1} \beta^{2} \int_{3 \bar{\varepsilon}_{0} / 4<r<(1-10 a / 9) \varepsilon_{0}} \bar{r}^{2-2 \beta} H^{2} r^{-1} d r d w
\end{aligned}
$$

Taking $\beta$ sufficiently large and using the growth hypothesis of Lemma 5.2 we may absorb the first term on the left into the right-hand side. Thus,

$$
\begin{aligned}
& c_{9}\left(\frac{1}{4} \bar{\varepsilon}_{0}\right)^{-2 \beta} \int_{\bar{\varepsilon}_{0} / 4<r<\bar{\varepsilon}_{0}} H^{2} r^{-1} d r d w \\
& \quad \geq \frac{1}{2} c_{1} \beta^{2} \int_{3 \bar{\varepsilon}_{0} / 4<r<(1-10 a / 9) \varepsilon_{0}} \bar{r}^{2-2 \beta} H^{2} r^{-1} d r d w .
\end{aligned}
$$

Fixing the choice of $\beta$ and relabeling our constants gives

$$
\int_{r<\bar{\varepsilon}_{0}} H^{2} r d r d w \geq c_{10}\left(\frac{\bar{\varepsilon}_{0}}{\varepsilon_{0}}\right)^{c_{11}} \int_{r<(1-4 a / 3) \varepsilon_{0}} H^{2} r d r d w .
$$

Here we used the obvious fact that the ball with radius $\bar{\varepsilon}_{0}$ includes the ball of radius $\frac{3}{4} \bar{\varepsilon}_{0}$. Lemma 5.2 follows upon replacing $H$ by its supremum in the integral of the left-hand side.

We conclude this phase of our argument with

Proposition 5.4. Suppose that $H$ satisfies the growth estimate for some $\varepsilon>\varepsilon_{0}$,

$$
\int_{(1-3 a / 2) \varepsilon<r<(1-a) \varepsilon} H^{2} \leq c_{2} \int_{(1-3 a) \varepsilon<r<(1-4 a / 3) \varepsilon} H^{2} .
$$

Then for all $0<\bar{\varepsilon}<\frac{\varepsilon}{100}$, we have

$$
\max _{r \leq \bar{\varepsilon}}|H|^{2} \geq c_{3}\left(\frac{\bar{\varepsilon}}{\varepsilon}\right)^{2 c_{4}} \operatorname{Av}_{r<(1-4 a / 3) \varepsilon}|H|^{2} .
$$

Proof. This follows by applying Lemma 5.2 to the function $H_{0}(x)=H\left(x \varepsilon / \varepsilon_{0}\right)$. Since $\varepsilon<\varepsilon_{0}$, the function $H_{0}$ satisfies the hypotheses of Lemma 5.2. The conclusion has been formulated to behave well under scaling. Of course, we interpret $\bar{\varepsilon}_{0}=\bar{\varepsilon} \varepsilon_{0} / \varepsilon$.

The next task is to relate the conclusion of Proposition 5.4 to nodal length. Suppose that $H$ is defined on the unit ball in $R^{2}$ and solves the equation 
$\Delta_{0} H=-\phi H$, where $\phi$ is bounded and smooth. In addition, assume that the lower bound (5.5) holds.

If $|z|<\bar{\varepsilon}$, then Taylor's formula with remainder implies, when $\bar{\varepsilon}<\frac{\varepsilon}{100}$,

$$
\left|H(z)-\sum_{|\alpha| \leq c_{4}} \frac{1}{\alpha !} \frac{\partial^{\alpha} H}{\partial z^{\alpha}}(0) z^{\alpha}\right| \leq \sup _{|z| \leq \bar{\varepsilon}|\alpha|=c_{4}+1} \sup _{2} b_{2}\left|\frac{\partial^{\alpha} H}{\partial z^{\alpha}}(z)\right| \bar{\varepsilon}^{c_{4}+1} .
$$

Here $\alpha=\left(\alpha_{1}, \alpha_{2}\right)$ and $\partial / \partial z^{\alpha}=\left(\partial / \partial x^{\alpha_{1}}\right)\left(\partial / \partial y^{\alpha_{2}}\right)$.

Employing standard elliptic theory to bound the normalized derivatives $\varepsilon^{\alpha}\left|\partial^{\alpha} H / \partial z^{\alpha}\right|$,

$$
\left|H(z)-\sum_{|\alpha| \leq c_{4}} \frac{1}{\alpha !} \frac{\partial^{\alpha} H}{\partial z^{\alpha}}(0) z^{\alpha}\right| \leq b_{3}\left(\underset{|z|<(1-4 a / 3) \varepsilon}{\operatorname{Av}|H|^{2}}\right)^{1 / 2}\left(\frac{\bar{\varepsilon}}{\varepsilon}\right)^{c_{4}+1} .
$$

Using (5.5), we get, for $|z|<\bar{\varepsilon}$,

$$
\left|H(z)-\sum_{|\alpha| \leq c_{4}} \frac{1}{\alpha !} \frac{\partial^{\alpha} H}{\partial z^{\alpha}}(0) z^{\alpha}\right| \leq b_{4}\left(\frac{\bar{\varepsilon}}{\varepsilon}\right) \max _{|z|<\bar{\varepsilon}}|H| .
$$

Taking $\bar{\varepsilon} / \varepsilon$ sufficiently small and using the triangle inequality yields

$$
\sup _{|\alpha| \leq c_{4}}\left|\frac{\partial^{\alpha} H}{\partial z^{\alpha}}(0)\right| \bar{\varepsilon}^{|\alpha|} \geq b_{5} \max _{|z| \leq \bar{\varepsilon}}|H| .
$$

We now fix the ratio $\frac{\bar{\varepsilon}}{\varepsilon}$ and return to (5.5) to deduce for $\varepsilon<\varepsilon_{0}$

$$
\sup _{|\alpha| \leq c_{4}}\left|\frac{\partial^{\alpha} H}{\partial z^{\alpha}}(0)\right| \varepsilon^{|\alpha|} \geq b_{6}\left(\underset{|z|<(1-4 a / 3 a) \varepsilon}{\operatorname{Av}}|H|^{2}\right)^{1 / 2} .
$$

Also, by standard elliptic theory, we may write

$$
\sup _{|z|<\varepsilon / 2} \sup _{|\alpha| \leq c_{4}+1} \varepsilon^{|\alpha|}\left|\frac{\partial^{\alpha} H}{\partial z^{\alpha}}(z)\right| \leq b_{7}\left(\underset{|z|<(1-4 a / 3) \varepsilon}{\operatorname{Av}}|H|^{2}\right)^{1 / 2} .
$$

The next step is to show that (5.6) and (5.7) imply a lower bound on the length of that part of the nodal set $N$ that is contained in a sufficiently small ball. It is convenient to develop this conclusion through a set of lemmas. The analogous one-dimensional result is

Lemma 5.8. Let $f \in C_{0}^{\infty}([-1,1])$. Suppose that

(i) $\max _{j \leq c_{12}}\left|f^{j}(0)\right| \geq b_{9}$.

(ii) $\max _{j \leq c_{12}+1} \max _{|x|<1}\left|f^{j}(x)\right| \leq b_{8}$.

Then $f$ has at most $c_{12}$ zeros in a sufficiently small interval $\left[-b_{10}, b_{10}\right]$.

Proof. If the conclusion fails, then by repeated applications of the mean value theorem, we find $\left|x_{j}\right| \leq b_{10}$ with $f^{j}\left(x_{j}\right)=0$ for any $j \leq c_{12}$. Integration and (ii) give $\left|f^{j}(0)\right| \leq b_{8} b_{10}$, contradicting (i) if $b_{10}$ is small. 
Suppose now that $g \in C^{\infty}(|z|<1)$ and $g$ satisfies

$$
\begin{aligned}
& \text { (i) } \sup _{|\alpha| \leq c_{4}}\left|\frac{\partial^{\alpha} g}{\partial z^{\alpha}}(0)\right| \geq b_{6} . \\
& \text { (ii) } \sup _{|z|<1 / 2} \sup _{|\alpha| \leq c_{4}+1}\left|\frac{\partial^{\alpha} g}{\partial z^{\alpha}}(z)\right| \leq b_{7} \text {. }
\end{aligned}
$$

We easily see that the following reformation of (5.9)(i) holds.

Lemma 5.10. For some $k \leq c_{4}$ and in a suitable rotation of the original coordinate system

$$
\left|\left(\frac{\partial}{\partial x}\right)^{k} g(0,0)\right| \geq b_{11} \text { and }\left|\left(\frac{\partial}{\partial y}\right)^{k} g(0,0)\right| \geq b_{11} .
$$

Proof. If the conclusion fails, then there exists a nonvanishing polynomial $P$ of order at most $c_{4}$ with

$$
\left|\left(\frac{\partial}{\partial x}\right)^{k} P(0,0)\right|=0 \text { or }\left|\left(\frac{\partial}{\partial y}\right)^{k} P(0,0)\right|=0
$$

for all $k \leq c_{4}$ and all rotations of our original coordinate system. However, if $P$ has a nonvanishing term of order $k$, then $v^{k} P(0,0)=0$ only for an isolated, and therefore finite, set of $v \in S^{1}$. There must be a pair $v_{1}$ and $v_{2}$ of orthogonal vectors with $v_{1}^{k} P(0,0) \neq 0$ and $v_{2}^{k} P(0,0) \neq 0$. This contradiction shows that $b_{11}$ exists.

The basic relationship between derivative estimates and nodal length in two dimensions is

Lemma 5.11. Let $g \in C^{\infty}(|z|<1)$ satisfy (5.9). Then the one-dimensional Hausdorff measure $\mathscr{H}^{1}$ of the set where $g(z)=0$ and $\nabla g(z) \neq 0$ satisfies

$$
\mathscr{H}^{1}\left\{z|| z \mid<b_{12}, g(z)=0, \nabla g(z) \neq 0\right\}<c_{13} .
$$

Proof. The set $\Gamma$ where $g(z)=0$ and $\nabla g(z) \neq 0$ is a one-dimensional manifold by the implicit function theorem. At each $z$, the associated tangent line makes an angle of at most $\frac{\pi}{4}$ with either the $x$-or $y$-axis. Therefore,

$$
\begin{aligned}
\mathscr{H}^{1}\left\{\Gamma \cap|z|<b_{12}\right\} \leq c_{14}\left[\int_{|s|<b_{12}}\right. & \mathscr{H}^{0}\left(\Gamma \cap x=s \cap|y|<b_{12}\right) d s \\
& \left.\quad+\int_{|s|<b_{12}} \mathscr{H}^{0}\left(\Gamma \cap y=s \cap|x|<b_{12}\right) d s\right] .
\end{aligned}
$$

Of course, $\mathscr{H}^{0}$ is merely the cardinality of the zero set. We assume that the $(x, y)$ coordinate system is chosen to satisfy the conclusion of Lemma 5.10.

If $b_{12}$ is sufficiently small, then we have

$$
\left|\left(\frac{\partial}{\partial y}\right)^{k} f(s, y)\right| \geq \frac{b_{11}}{2}
$$


for each $|s|<b_{12}$. This is a consequence of Lemma 5.10 and hypotheses (5.9)(ii). By Lemma 5.8, we get

$$
\mathscr{H}^{0}\left(\Gamma \cap x=s \cap|y|<b_{12}\right)<b_{14} .
$$

A similar argument applies to the second integral in (5.12). This completes the proof of Lemma 5.11 .

Dividing $H$ by its average $\left(\operatorname{Av}_{|z|<(1-4 a / 3) \varepsilon}|H|^{2}\right)^{1 / 2}$ does not change its zero set, and so rescaling also to the unit ball gives a function $g$ satisfying (5.9). We apply Lemma 5.11 and return to scale $\varepsilon$ concluding

Proposition 5.13. Let $H$ be a solution of $\Delta_{0} H=-\phi H$. Suppose that $H$ satisfies (5.6) and (5.7). Then

$$
\mathscr{H}^{1}\left(z|| z \mid<b_{15} \varepsilon \text { and } H(z)=0\right)<c_{15} \varepsilon .
$$

Note that the nodal set of $H$ contains only a finite number of singular points in any compact subset of its domain of definition. Therefore, we need not require $\nabla H \neq 0$ in the conclusion of Proposition 5.13.

For later reference, we summarize the principal conclusion of this section in

Proposition 5.14. Let $H$ be defined in a Euclidean unit ball and solve $\Delta_{0} H=$ $-\phi H$. Suppose that for some $\varepsilon \leq \varepsilon_{0} H$ satisfies the growth estimate

$$
\int_{(1-3 a / 2) \varepsilon<r<(1-a) \varepsilon} H^{2} \leq c_{2} \int_{(1-3 a) \varepsilon<r<(1-4 a / 3) \varepsilon} H^{2} .
$$

Then

$$
\mathscr{H}^{1}\left\{z|| z \mid<b_{15} \varepsilon \text { and } H(z)=0\right\}<c_{15} \varepsilon .
$$

We note that Propositions 5.4 and 5.14 have the same hypotheses. The conclusion of Proposition 5.4 was used to establish (5.6) and (5.7). Using Proposition 5.13, we deduce the result of Proposition 5.14.

\section{TOTAL NODAL LENGTH}

It is now a straightforward matter to complete the proof for Theorem 1.2. We have shown that our eigenfunctions cannot grow rapidly on too many small balls. Moreover, an upper bound on growth of $L^{2}$ norm has imposed an upper bound on the local nodal length. We must merge these two ingredients. For this purpose, we rely upon a process of repeated subdivision and selection of squares. This method is reminiscent of the techniques employed by Calderon and Zygmund in their study of singular integral operators [11].

Let $M$ be a compact closed Riemannian surface. We will discuss only the detailed proof of Theorem 1.2 for $\partial M$ empty. If $\partial M \neq \varnothing$, we employ the doubling approach of [6] along with small modifications of the present proof. Suppose that $\Delta F=-\lambda F$, so that $F \in C^{\infty} M$ is an eigenfunction of the canonical Laplacian associated to the Riemannian metric of $M$. Assume that $B\left(p, c_{1} \lambda^{-1 / 4}\right)$ is a geodesic ball of $M$. If $c_{1}$ is sufficiently small, then this ball 
will be contained in an isothermal coordinate patch. Consequently, we have $\Delta_{0} F=-\lambda \phi_{1} F$, where $\Delta_{0}$ is the Euclidean Laplacian.

Define $G(z)=F\left(c_{2} \lambda^{-1 / 4} z\right)$. If $c_{2}$ is sufficiently small, then $G$ is defined on a Euclidean ball of radius three centered at the origin in $R^{2}$. Moreover, $\Delta_{0} G=-\lambda^{1 / 2} \phi_{2} G$, where we may assume that $\phi_{2}$ is sufficiently small. Divide $\left\{x, y \mid \max (|x|,|y|) \leq \frac{1}{60}\right\}$ into a grid of closed squares $P_{i}$ each of side $\delta \leq$ $b_{1} \lambda^{-1 / 2}$. If equation (4.6) holds for some sufficiently large $c_{13}$ and for some $z_{\nu} \in P_{\nu}$, we say that $P_{\nu}$ is a square of rapid growth. We apply Proposition 4.7 to prove

Lemma 6.1. There are at most $c_{3} \lambda$ squares of side $\delta$ where $G$ has rapid growth. Proof. Let $I_{1}$ be the collection of those indices $i$ for which $P_{i}$ is a square of rapid growth. For each $\nu \in I_{1}$, choose a point $z_{\nu} \in P_{\nu}$ where (4.6) holds. Define $D_{\nu}^{*}=\left\{z|| z-z_{\nu} \mid<b_{2} \delta \lambda^{1 / 4}\right\}$. The collection of disks $D_{\nu}^{*}$ covers the collection of squares $P_{\nu}$ for $\nu \in I_{1}$. Let $I_{2} \subset I_{1}$ be an indexing set for a maximal collection of disjoint $D_{\nu}^{*}$. If $\nu \in I_{2}$, then define $D_{\nu}^{* *}=\left\{z|| z-z_{\nu} \mid<\right.$ $\left.4 b_{2} \delta \lambda^{1 / 4}\right\}$. By maximality of $I_{2}$, we deduce that

$$
\bigcup_{\nu \in I_{2}} D_{\nu}^{* *} \supseteq \bigcup_{\nu \in I_{1}} D_{\nu}^{*} \supset \bigcup_{\nu \in I_{1}} P_{\nu}
$$

Thus $\left|I_{2}\right| \times 16 b_{2}^{2} \delta^{2} \lambda^{1 / 2} \geq\left|I_{1}\right| \delta^{2}$, where $\left|I_{1}\right|$ denotes the cardinality of $I_{1}$. So $\left|I_{2}\right| \lambda^{1 / 2} \geq b_{3}\left|I_{1}\right|$.

However, Proposition 4.7 implies that $\left|I_{2}\right| \leq b_{4} \lambda^{1 / 2}$. This combines with our previous observations to give the required estimate $\left|I_{1}\right| \leq c_{3} \lambda$.

We now introduce a process of controlled bisection. Begin by dividing $\left\{(x, y) \mid \max (|x|,|y|) \leq \frac{1}{60}\right\}$ into a grid of squares $P_{i}(1)$ of side $\delta(1)=b_{1} \lambda^{-1 / 2}$. Separate the collection $P_{i}(1)$ into those squares $Q_{j}(1)$, where $G$ has rapid growth, and $R_{l}(1)$, where $G$ has slow growth. Of course, slow growth means that (4.6) fails for each point $z_{\nu} \in R_{l}(1)$. Each square $Q_{j}(1)$ of rapid growth is bisected to obtain squares $P_{i}(2)$ of side $\delta(2)=\delta(1) / 2$. Again, the collection $P_{i}(2)$ may be grouped into the subcollections $Q_{j}(2)$ and $R_{j}(2)$ of rapid and slow growth. Proceeding inductively, at each stage bisecting only those squares of rapid growth, we have squares $Q_{j}(k)$ and $R_{j}(k)$ of side $\delta(k)=\delta(1) / 2^{k}$. It is easy to deduce

Lemma 6.2. For each fixed $k \geq 1$,

(i) There are at most $c_{3} \lambda$ squares $Q_{j}(k)$ with rapid growth.

(ii) There are at most $c_{4} \lambda$ squares $R_{l}(k)$ with slow growth.

Proof. (i) is an immediate consequence of Lemma 6.1. If $k=1$, (ii) holds simply because the total number of all squares is at most the order of $\lambda$. When $k \geq 2$, each $R_{l}(k)$ is obtained by bisecting some $Q_{j}(k-1)$, so (ii) follows from (i). 
Almost every point will eventually lie in some good square $R_{l}(k)$. In fact, the stronger

Lemma 6.3. $\bigcup_{k, l} R_{l}(k)$ covers $\left\{(x, y) \mid \max (|x|,|y|) \leq \frac{1}{60}\right\}$ except for finitely many points.

Proof. First suppose that $G\left(x_{0}, y_{0}\right) \neq 0$ and $\left(x_{0}, y_{0}\right) \in P_{i}(k)$ with a sufficiently large $k$. Since $G$ is a continuous function, it will be nearly constant on $P_{i}(k)$. Thus, (4.6) must fail for $\delta=\delta(k)$ and any $z_{\nu} \in P_{i}(k)$. Recall that we assumed throughout that the constant $c_{13}$ of equation (4.6) is suitably large.

Now assume that $G\left(x_{0}, y_{0}\right)=0$ but $d G\left(x_{0}, y_{0}\right) \neq 0$. Then the implicit function theorem provides a local change of variables so that $G$ is a coordinate function $\bar{x}$ near $\left(x_{0}, y_{0}\right)$. It is an elementary exercise in calculus and measure theory to see that (4.6) must fail for such coordinate functions $\bar{x}$ when $\delta=\delta(k)$ is sufficiently small.

So if $\left(x_{0}, y_{0}\right)$ does not lie in some square $R_{l}(k)$ of slow growth, it must be one of the finitely many singular points where $G\left(x_{0}, y_{0}\right)=0$ and $d G\left(x_{0}, y_{0}\right)=$ 0 .

The last ingredient required for the proof of Theorem 1.2 is

Lemma 6.4. $\mathscr{H}^{1}\left(z \mid G(z)=0\right.$ and $\left.z \in R_{l}(k)\right)<c_{5} 2^{-k} \lambda^{-1 / 2}$ for each fixed $k$ and $l$.

Proof. This follows by applying Proposition 5.14 with $H(z)=G\left(z_{\nu}+\varepsilon_{0}^{-1} \delta(k) z\right)$ and a suitable finite collection of $z_{\nu} \in R_{l}(k)$. We rescale to obtain Lemma 6.4.

From Lemma 6.3,we have

$$
\begin{gathered}
\mathscr{H}^{1}\left(z \mid G(z)=0 \text { and } \max (|x|,|y|) \leq \frac{1}{60}\right) \\
\quad \leq \sum_{k, l} \mathscr{H}^{1}\left(z \mid G(z)=0 \text { and } z \in R_{l}(k)\right) .
\end{gathered}
$$

The sum on the right-hand side is estimated via Lemma 6.2(ii) and Lemma 6.4 to yield

$$
\mathscr{H}^{1}\left(z \mid G(z)=0 \text { and } \max (|x|,|y|) \leq \frac{1}{60}\right) \leq c_{4} \lambda \sum_{k} c_{5} 2^{-k} \lambda^{-1 / 2} \leq c_{6} \lambda^{1 / 2} .
$$

Since $G(z)=F\left(c_{2} \lambda^{-1 / 4} z\right)$, we deduce Theorem 1.2 via multiplication by the scaling factor $\lambda^{-1 / 4}$.

\section{BIBLIOGRAPHY}

1. N. Aronszajn, A unique continuation theorem for solutions of elliptic partial differential equations of second order, J. Math. Pures Appl. 36 (1957), 235-249.

2. J Brüning, Uber knoten von eigenfunktionen des Laplace-Beltrami operators, Math. Z. 158 (1978), 15-21.

3. T. Carleman, Sur les systémes linéares aux dérivées partielles du premier ordre á deux variables, C. R. Acad. Sci. Paris Ser. I Math. 197 (1933), 471-474.

4. J. Cheeger, Finiteness theorems in Riemannian geometry, Amer. J. Math. 92 (1970), 61-74. 
5. H. Donnelly and C. Fefferman, Nodal sets of eigenfunctions on Riemannian manifolds, Invent. Math. 93 (1988), 161-183.

6. __ Nodal sets of eigenfunctions: Riemannian manifolds with boundary, Moser volume, Analysis, Academic Press, New York, 1990, pp. 251-262.

7. S. Goldberg, Curvature and homology, Dover, New York, 1962.

8. R. S. Hardt and L. Simon, Nodal sets of solutions of elliptic equations, J. Differential Geom. 30 (1989), 505-522.

9. J. Jost, Harmonic maps between surfaces, Lecture Notes in Math., vol 1062, Springer-Verlag, Berlin, Heidelberg, and New York, 1984.

10. N. S. Nadirashvili, Length of nodal sets of eigenfunctions of the Laplace operator, Uspekhi Mat. Nauk 43 (1988), 219-220.

11. E. M. Stein, Singular integrals and differentiability properties of functions, Princeton University Press, Princeton, NJ, 1970.

Department of Mathematics, Purdue University, West Lafayette, Indiana 47907

Department of Mathematics, Princeton University, Princeton, New Jersey 08544 